\title{
Avaliação dos intervalos de referência de lípides e lipoproteinas para crianças e adolescentes: associação das dislipidemias e sobrepeso em escolares de 4 a 19 anos de idade de Maracaí (São Paulo)
}

\author{
Evaluation of lipids and lipoproteins range values for children and adolescents: associations of dyslipidemia to \\ overweight in schoolchildren from 4 to 19 years old from Maracaí (State of São Paulo)
}

Mario Seki ${ }^{1}$; Ana Maria Bonametti ${ }^{2}$; Tiemi Matsuo ${ }^{3}$; Marisa Okabe Seki ${ }^{4}$; Mariana de Rezende Andreghettij; Alexandre José Faria Carrilho ${ }^{2}$

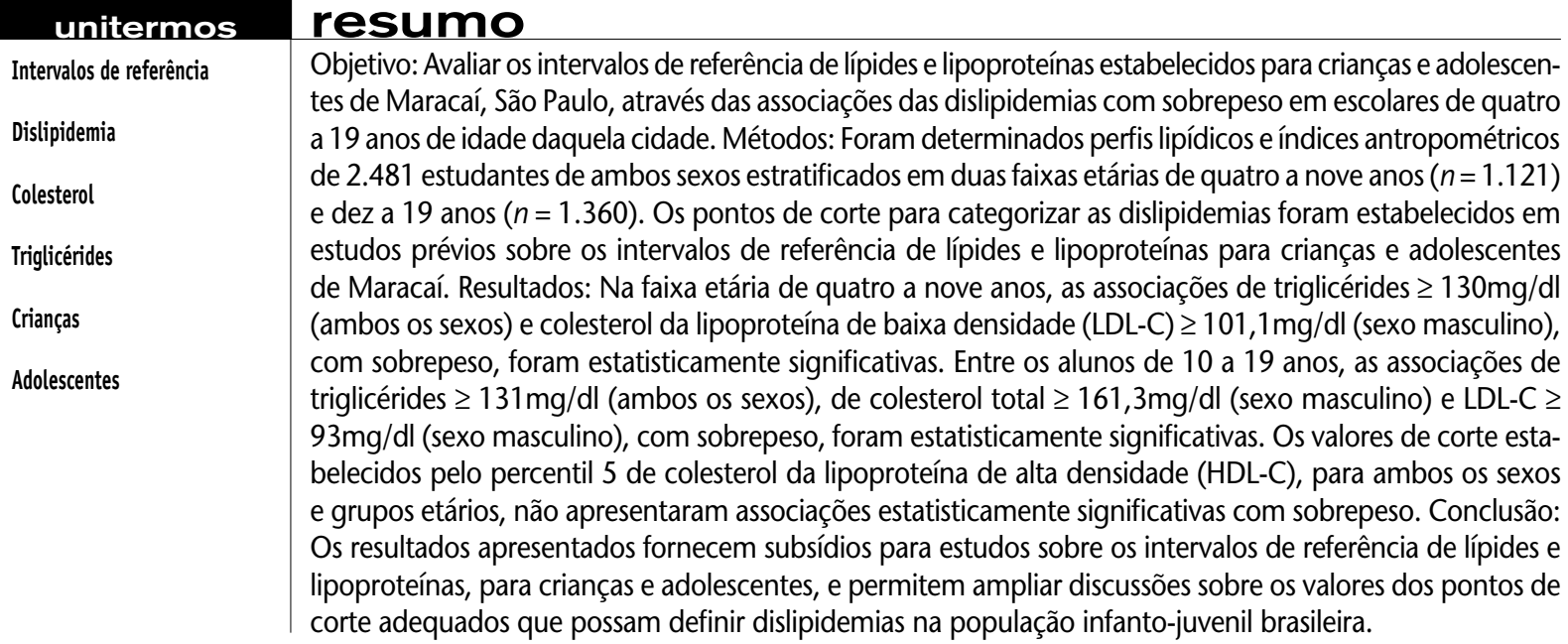

abstract

Objective: To evaluate the lipids and lipoproteins range values established for children and adolescents from Maracaí, State of São Paulo, through the associations of dyslipidemia and overweight in students from four to 19 years old, from that city. Methods: The lipid profiles and anthropometric indexes were determined for 2,481 students of both sexes, separated into two age ranges from four to nine $(n=1,121)$ and ten to $19(n=1,360)$. The cut-points to rank the dyslipidemia have been set up in previous works on range values for lipids and lipoproteins for children and adolescents from Maracaí, State of São Paulo. Results: In the four to nine years old, the associations of triglycerides $\geq$ $130 \mathrm{mg} / \mathrm{dl}$ (for both sexes) and of LDL-cholesterol $\geq 101.1 \mathrm{mg} / \mathrm{dl}$ (male) to overweight were statistically significant. Among the students from ten to 19 years old, the associations of triglyceride $\geq 131 \mathrm{mg} / \mathrm{dl}$ (both sexes), of total cholesterol $\geq 161.3 \mathrm{mg} / \mathrm{dl}$ (male) and LDL-cholesterol $\geq 93 \mathrm{mg} / \mathrm{dl}$ (male) were statistically significant. The cut-points established by percentile 5 of HDL-cholesterol for both sexes and age group did not show significant associations to overweight. Conclusion: The achieved results supply evidence for studies on the range values for lipids and lipoproteins for children and adolescents, and allow for broadening the discussions on the values for reasonable cut-points that could define dyslipidemia within the Brazilian population of children and youngsters. key words

Reference interval

Dyslipidemia

Cholesterol

Triglycerides

Children

Adolescents

\footnotetext{
1. Médico patologista clínico; mestre em Medicina e Ciências da Saúde da Universidade Estadual de Londrina (UEL); aluno do Programa de Pós-Graduação em Medicina e Ciências da Saúde (doutorado) da UEL.

2. Departamento de Clínica Médica do Centro de Ciências da Saúde da UEL.

3. Departamento de Estatística e Matemática Aplicada da UEL.

4. Médica residente do Serviço de Laboratório Clínico da Santa Casa de São Paulo (SCSP).

5. Estudante de Medicina da Universidade de Santo Amaro (UNISA).

Parte integrante da dissertação de mestrado apresentada ao Programa de Pós-Graduação em Medicina e Ciências da Saúde da UEL, em dezembro de 2002, com o título Perfil lipídico em escolares de dois a 19 anos de idade da cidade de Maracaí (SP): aspectos epidemiológicos e estudo dos intervalos de referência.
} 


\section{Introdução}

A prevenção das doenças cardiovasculares (DCV) deve ser iniciada na infância e na adolescência, com avaliação do perfil lipídico e de outros fatores de risco( ${ }^{(9)}$. Em adultos, os valores dos pontos de corte para lípides e lipoproteínas para diagnóstico e tratamento estão bem estabelecidos pelas III Diretrizes Brasileira Sobre Dislipidemias (DBSD) ${ }^{(11)}$.

Apesar da relevância das dislipidemias na prevenção das DCV, ainda são raros os estudos sobre os pontos de corte e os valores adequados de lípides e lipoproteínas que possam definir as necessidades de intervenções individuais em crianças e adolescentes brasileiros. As DBSD ${ }^{(11)}$ adotam os valores do National Cholesterol Education Program $(\text { NCEP })^{(9)}$ estabelecidos para crianças e adolescentes dos EUA. Esses valores, que foram adotados para crianças $\mathrm{e}$ adolescentes brasileiros, não consideraram as diferenças com a população norte-americana nas múltiplas variáveis que interferem nas concentrações de lípides e lipoproteínas, como hábitos alimentares ${ }^{(2,9)}$ e heranças genéticas ${ }^{(9,11)}$.

Anormalidades genéticas no mecanismo de uma ou mais lipoproteínas podem produzir aumento nos níveis de colesterol total (CT), triglicérides (TG) e/ou de ambos ${ }^{(9)}$. Apesar disso, os fatores críticos que influenciam a elevação de CT em muitos indivíduos são os hábitos de ingestão alimentar, com excesso de quantidade de ácidos graxos saturados, de CT e de energia total ${ }^{(9)}$.

Há uma distribuição desigual das concentrações médias de lípides e lipoproteínas em crianças e adolescentes de populações diferentes (2). Por exemplo, os valores médios de CT são maiores nos caucasianos e norte-americanos que nos orientais e africanos ${ }^{(2)}$. Além disso, há uma grande variação entre os níveis de $\mathrm{CT}$, em relação à idade, ao sexo e à raça em indivíduos de uma mesma população(2).

Mais recentemente, a I Diretriz da Prevenção da Aterosclerose na Infância e Adolescência (DPAIA) propôs valores de referência de lípides e lipoproteínas para crianças e adolescentes brasileiros ${ }^{(5)}$. Pelos critérios adotados pela DPAIA, a classe de recomendação é de existência de divergência sobre a indicação com divisão de opiniões, enquanto o nível de evidência é de publicações de consensos e opiniões de especialistas ${ }^{(5)}$.

Os estudos sobre os valores dos pontos de corte obtidos através dos intervalos de referência para crianças ${ }^{(12)} \mathrm{e}$ adolescentes ${ }^{(13)}$ de Maracaí (SP) apresentaram valores divergentes dos adotados pelas DBSD ${ }^{(11)}$. Os valores de Maracaí (SP), entretanto, não foram validados com critérios clínicos consistentes e de interesse epidemiológico.
O objetivo deste estudo é avaliar os pontos de corte dos intervalos de referência de lípides e lipoproteínas estabelecidos para crianças e adolescentes de Maracaí, através das associações das dislipidemias com sobrepeso em escolares de quatro a 19 anos dessa cidade.

\section{Casuística e métodos}

Este estudo transversal, aprovado pelo Comitê de Bioética em Pesquisa da Universidade Estadual de Londrina (UEL), foi realizado no período de março a outubro de 2002, em Maracaí, município localizado no sudoeste do Estado de São Paulo, com uma população de 12.438 habitantes, segundo o Censo de 2000 do Instituto Brasileiro de Geografia e Estatística (IBGE).

O processo de amostragem do estudo considerou como critérios de inclusão uma população elegível de 3.189 escolares de ambos os sexos, aparentemente saudáveis, matriculados no pré-escolar, e nas escolas de ensino fundamental e médio de Maracaí. Oito escolas (sete públicas e uma particular), ou seja, $100 \%$ das instituições de ensino da cidade, localizadas nas zonas urbana e rural, aceitaram os convites para participar do estudo.

Os alunos foram aceitos como voluntários mediante autorização do pai e/ou responsável, conforme termo de consentimento livre e esclarecido. Foram adotados os seguintes critérios de exclusão: escolares com sintomatologias evidentes de morbidades severas, com histórico de dislipidemias secundárias a doenças ${ }^{(11)}$, em uso de medicamentos que interferem nas concentrações de lípides e lipoproteínas ${ }^{(11)}$, e com concentrações de TG $\geq 400 \mathrm{mg} / \mathrm{dl}$, que prejudicam os cálculos dos valores de colesterol da lipoproteína de baixa densidade (LDL-C), segundo as DBSD ${ }^{(11)}$.

Foram realizadas reuniões preparatórias para orientar os pais e/ou responsáveis dos alunos sobre a importância da prevenção das DCV na infância e adolescência, e as condições ideais para obtenção dos dados físicos. Os cuidados para coleta de materiais biológicos, incluindo jejum prévio de 12 a 14 horas, seguiram recomendações das $\operatorname{DBSD}^{(11)}$. Os cadastros individuais, históricos pessoais e informações sobre antecedentes familiares, foram obtidos com a colaboração de professores e inspetores de ensino treinados para essa finalidade.

As medidas antropométricas foram obtidas por auxiliares de enfermagem registrados no Conselho Regional de Enfermagem (COREN). Os alunos, vestindo roupas leves, foram pesados e medidos descalços em balanças e antropômetros 
da marca Filizola, aferidos pelo Instituto de Pesos e Medidas (IPM). Foram tomadas duas medidas para peso e duas para altura, extraindo-se a média aritmética simples. O intervalo das medições foi de $0,1 \mathrm{~kg}$ para peso e $1 \mathrm{~cm}$ para altura. Para avaliar o estado nutricional em escolares até nove anos de idade, optou-se pelo escore Z para peso e altura (WHZ). Por esse método, de acordo com a Organização Mundial da Saúde (OMS), são considerados como sobrepeso os valores de $Z \geq+2$ desvios padrão (+ $2 \mathrm{DP})^{(17)}$.

No grupo de 10 a 19 anos foram utilizados valores para população brasileira, segundo Sichieri e Allam ${ }^{(14)}$, que classificam na categoria de sobrepeso os adolescentes com índice de massa corpórea (IMC) igual ou maior ao percentil 90 ajustados para idade e sexo.

Os materiais biológicos colhidos nas escolas de Maracaí por auxiliares de laboratório habilitados para essa finalidade seguiram os cuidados pré-analíticos recomendados pelas DBSD ${ }^{(11)}$. O processamento do sangue total para obtenção do soro, conservação e transporte dos materiais seguiram os procedimentos operacionais-padrão do Laboratório Dr. Joelson (Assis-SP) e do Labmed/Labimagem (Londrina-PR), ambos certificados em sistema da qualidade ISO desde 1999.

As determinações bioquímicas para reações enzimáticas de TG, CT e colesterol da lipoproteína de alta densidade (HDL-C) foram realizadas no Labmed/Labimagem, que utilizou kits de química seca Johnson \& Johnson e autoanalisador Vitros 750. A metodologia laboratorial foi detalhada em estudos publicados anteriormente ${ }^{(12,13)}$. A fração LDL-C, obtida pelo cálculo de Friedewald: LDL-C = CT - HDL-C - TG/5, é recomendada para concentrações de TG abaixo de $400 \mathrm{mg} / \mathrm{dl}^{(5,11)}$.

Os controles de qualidade internos para as variáveis bioquímicas deste estudo foram realizados diariamente com soros-controles do fabricante. Os controles externos da qualidade foram realizados com o Programa de Excelência para Laboratórios Médicos da Sociedade Brasileira de Patologia Clínica (PELM/SBPC), que apresentaram ótimos desempenhos durante o ano de 2002 para TG, CT e HDL-C.

Para categorizar as dislipidemias, foram adotados os pontos de corte estabelecidos pelos intervalos de referência de lípides e lipoproteínas para escolares até nove anos ${ }^{(12)}$, e para os de dez a 19 anos ${ }^{(13)}$. Esses valores foram obtidos através dos percentil 95 (P95) para TG, percentil 75 (P75) para CT e LDL-C, e percentil 5 (P5) para HDL-C. A definição dos percentis que estabelecem os valores dos pontos de corte é a mesma adotada pelo Lipids Research Clinics Program (LRCP) $)^{(10)}$ e pelo NCEP ${ }^{(9)}$.
Os dados foram armazenados no programa Excel (versão 2000) e analisados no Epilnfo versão 6.04d, Center for Diseases Control and Prevention (CDC), Atlanta, em 2001, com testes do $\chi^{2}$ com correção de Yates para os casos em que todas as freqüências esperadas foram superiores a cinco ou, caso contrário, empregou-se o teste exato de Fisher. A amostra mínima foi calculada para uma freqüência esperada de $35 \%$ de alterações de $\mathrm{CT}^{(8)}$ e um erro máximo de $1 \%$.

\section{Resultados}

Do total de 3.189 escolares matriculados em Maracaí, foram avaliados dados de 2.528 alunos de ambos os sexos. As 47 exclusões foram devidas aos critérios previamente estabelecidos, entre os quais dois alunos (ambas as faixas etárias) que apresentaram valores de TG $\geq 400 \mathrm{mg} / \mathrm{dl}$. As 661 perdas foram referentes aos alunos matriculados que não participaram do estudo, a dados que apresentaram irregularidades de preenchimento no protocolo (cadastros individuais, históricos pessoais, etc.), a coletas inadequadas das medidas antropométricas e/ou a exames laboratoriais.

O tamanho da amostra ( $n$ ) de 2.481, que corresponde a $77,8 \%$ da população escolar matriculada em Maracaí, foi superior ao mínimo estimado para este estudo de 2.313 indivíduos. A amostra dos alunos de quatro a nove anos $(n=$ 1.121), que representou $89,9 \%$ dos escolares matriculados nas escolas de Maracaí (SP) nessa faixa etária, foi constituída por $536(47,8 \%)$ do sexo feminino e 585 (52,2\%) do masculino. A média de idade das crianças participantes foi de 7,2 $\pm 1,5$ e mediana de 7,2 anos. Entre os alunos de dez a 19 anos, a participação $(n=1.360)$ correspondeu a $70 \%$ dos estudantes matriculados nessa faixa etária, dos quais 702 foram do sexo feminino (51,6\%) e 658 do masculino (48,4\%). A idade média correspondente foi de 12,9 $\pm 2,1$ e mediana de 13 anos.

Foi observada uma prevalência de sobrepeso ( $Z \geq+2$ DP) de $9,6 \%$ em escolares de quatro a nove anos, e 10,2\% (IMC $\geq$ P90) entre os alunos de 10 a 19 anos.

As associações de TG $\geq$ P95 (ambos os sexos) e de LDL-C $\geq$ P75 (sexo masculino) foram estatisticamente significativas com sobrepeso em escolares de quatro a nove anos (Tabela 1). Entre os alunos de 10 a 19 anos, as associações com sobrepeso foram estatisticamente significativas para TG $\geq$ P95 (ambos os sexos), e CT e LDL-C $\geq$ P75 (sexo masculino) com sobrepeso (Tabela 2).

As associações de HDL-C $\leq$ P5 e sobrepeso não foram estatisticamente significativas em ambas as faixas etárias e sexos (Tabelas 1 e 2). 
Distribuiçáo de escolares de 4 a 9 anos de idade conforme resultados de lípides e

Tabela 1 lipoproteínas, sexo e estado nutricional (escore Z) - Maracai-SP, 2002

\begin{tabular}{|c|c|c|c|c|c|c|c|c|c|c|c|c|c|c|c|}
\hline \multirow{4}{*}{$\begin{array}{l}\text { Lipides e } \\
\text { lipoproteínas } \\
\text { (mg/dl) }\end{array}$} & \multicolumn{15}{|c|}{ Sexo e estado nutricional } \\
\hline & \multicolumn{8}{|c|}{ Feminino } & \multicolumn{7}{|c|}{ Masculino } \\
\hline & \multicolumn{2}{|c|}{$\mathrm{Z} \geq+2 \mathrm{DP}$} & \multicolumn{2}{|c|}{$\mathrm{Z}<+2 \mathrm{DP}$} & \multicolumn{2}{|c|}{ Total } & \multirow{2}{*}{$\chi^{2}$} & \multirow{2}{*}{$p$} & \multicolumn{2}{|c|}{$\mathrm{Z} \geq+2 \mathrm{DP}$} & \multicolumn{2}{|c|}{$\mathrm{Z}<+2 \mathrm{DP}$} & \multicolumn{2}{|c|}{ Total } & \multirow{2}{*}{$\chi^{2} \quad p$} \\
\hline & $n$ & $\%$ & $n$ & $\%$ & $n$ & $\%$ & & & $n$ & $\%$ & $n$ & $\%$ & $n$ & $\%$ & \\
\hline $\mathrm{TG} \geq 130$ & 8 & 18,2 & 27 & 5,5 & 35 & 6,5 & \multicolumn{2}{|c|}{$\begin{array}{c}\text { Teste exato } \\
\text { de Fisher } \\
=0,004\end{array}$} & 10 & 15,6 & 21 & 4 & 31 & 5,3 & $\begin{array}{c}\text { Teste exato } \\
\text { de Fisher } \\
<0,001\end{array}$ \\
\hline $\mathrm{TG}<130$ & 36 & 81,8 & 465 & 94,5 & 501 & 93,5 & & & 54 & 84,4 & 500 & 96 & 554 & 94,7 & \\
\hline $\mathrm{CT} \geq 170$ & 14 & 31,8 & 134 & 27,2 & 148 & 27,6 & \multirow{2}{*}{0,23} & \multirow{2}{*}{0,634} & 19 & 29,7 & 124 & 23,8 & 143 & 24,4 & \multirow{2}{*}{0,77} \\
\hline $\mathrm{CT}<170$ & 30 & 68,2 & 358 & 72,8 & 388 & 72,4 & & & 45 & 70,3 & 397 & 76,2 & 442 & 75,6 & \\
\hline LDL-C $\geq 101,1$ & 17 & 38,6 & 128 & 26,0 & 145 & 27,1 & \multirow{2}{*}{2,65} & \multirow{2}{*}{0,103} & 27 & 42,2 & 129 & 24,8 & 156 & 26,7 & \multirow{2}{*}{7,98} \\
\hline $\mathrm{LDL}<101,1$ & 27 & 61,4 & 364 & 74,0 & 391 & 72,9 & & & 37 & 57,8 & 392 & 75,2 & 429 & 73,3 & \\
\hline HDL-C $\leq 34$ & 6 & 13,6 & 33 & 6,7 & 39 & 7,3 & \multirow{2}{*}{\multicolumn{2}{|c|}{$\begin{array}{c}\text { Teste exato } \\
\text { de Fisher = } \\
0,119\end{array}$}} & 4 & 6,3 & 34 & 6,5 & 38 & 6,5 & \multirow[t]{2}{*}{$\begin{array}{l}\text { Teste exato } \\
\text { de Fisher = } 1\end{array}$} \\
\hline HDL-C $>34$ & 38 & 86,4 & 459 & 93,3 & 497 & 92,7 & & & 60 & 93,8 & 487 & 93,5 & 547 & 93,5 & \\
\hline
\end{tabular}

\section{Distribuição de escolares de 10 a 19 anos de idade conforme resultados de lípides e} Tabela 2 lipoproteinas, sexo e estado nutricional (IMC) - Maracai-SP, 2002

\begin{tabular}{|c|c|c|c|c|c|c|c|c|c|c|c|c|c|c|c|c|}
\hline \multirow{4}{*}{$\begin{array}{l}\text { Lipides e } \\
\text { lipoproteínas } \\
\text { (mg/dl) }\end{array}$} & \multicolumn{16}{|c|}{ Sexo e estado nutricional } \\
\hline & \multicolumn{8}{|c|}{ Feminino } & \multicolumn{8}{|c|}{ Masculino } \\
\hline & \multicolumn{2}{|c|}{$\mathrm{IMC} \geq \mathrm{P} 90$} & \multicolumn{2}{|c|}{$\mathrm{IMC}<\mathrm{P} 90$} & \multicolumn{2}{|c|}{ Total } & \multirow[b]{2}{*}{$\chi^{2}$} & \multirow[b]{2}{*}{$p$} & \multicolumn{2}{|c|}{ IMC $\geq \mathrm{P} 90$} & \multicolumn{2}{|c|}{$\mathrm{IMC}<\mathrm{P} 90$} & \multicolumn{2}{|c|}{ Total } & \multirow[b]{2}{*}{$\chi^{2}$} & \multirow[b]{2}{*}{$p$} \\
\hline & $n$ & $\%$ & $n$ & $\%$ & $n$ & $\%$ & & & $n$ & $\%$ & $n$ & $\%$ & $n$ & $\%$ & & \\
\hline $\mathrm{TG} \geq 131$ & 10 & 15,2 & 41 & 6,4 & 51 & 51 & \multicolumn{2}{|c|}{$\begin{array}{l}\text { Teste exato } \\
\text { de Fisher } \\
=0,022\end{array}$} & 17 & 23,3 & 32 & 5,5 & 49 & 7,4 & \multirow[t]{2}{*}{27,37} & $<0,001$ \\
\hline $\mathrm{TG}<131$ & 56 & 84,8 & 595 & 93,6 & 651 & 92,7 & & & 56 & 76,7 & 553 & 94,5 & 609 & 92,6 & & \\
\hline $\mathrm{CT} \geq 161,3$ & 22 & 33,3 & 181 & 28,5 & 203 & 28,9 & \multirow{2}{*}{0,45} & \multirow{2}{*}{0,491} & 28 & 38,4 & 124 & 21,2 & 152 & 23,1 & \multirow{2}{*}{9,81} & \multirow{2}{*}{0,001} \\
\hline CT $<161,3$ & 44 & 66,7 & 455 & 71,5 & 499 & 71,1 & & & 45 & 61,6 & 461 & 78,8 & 506 & 76,9 & & \\
\hline LDL-C $\geq 93$ & 26 & 39,4 & 174 & 27,4 & 200 & 28,5 & \multirow[b]{2}{*}{3,68} & \multirow{2}{*}{0,055} & 29 & 39,7 & 128 & 21,9 & 157 & 23,9 & \multirow{2}{*}{10,42} & \multirow[b]{2}{*}{0,001} \\
\hline LDL-C $<93$ & 40 & 60,6 & 462 & 72,6 & 502 & 71,5 & & & 44 & 60,3 & 457 & 78,1 & 501 & 76,1 & & \\
\hline HDL-C $\leq 33$ & 2 & 3,0 & 34 & 5,3 & 36 & 5,1 & \multirow{2}{*}{\multicolumn{2}{|c|}{$\begin{array}{l}\text { Teste exato } \\
\text { de Fisher } \\
=0,565\end{array}$}} & 4 & 5,5 & 30 & 5,1 & 34 & 5,2 & \multirow{2}{*}{\multicolumn{2}{|c|}{$\begin{array}{l}\text { Teste exato } \\
\text { de Fisher } \\
=0,783\end{array}$}} \\
\hline HDL-C > 33 & 64 & 97 & 602 & 94,7 & 666 & 94,9 & & & 69 & 94,5 & 555 & 94,9 & 624 & 94,8 & & \\
\hline
\end{tabular}

\section{Discussão}

O conceito atual sobre valores de referência para lípides representa uma exceção nos princípios habitualmente adotados em Medicina Laboratorial e tem por objetivo definir os limites que pressupõem a necessidade de se discriminar indivíduos com elevados níveis de lipoproteínas no soro dos normais ${ }^{(1)}$.

Os critérios que definem os valores alterados de lípides e lipoproteínas na infância e na adolescência são obtidos em percentis previamente estabelecidos ${ }^{(1,9,10)}$. Para definir valores de referência baseados em percentis, é necessário estabelecer a distribuição dos níveis relevantes de lípides e lipoproteínas através de estudos em uma amostra de população bem definida ${ }^{(10)}$.

O P75 para CT e LDL-C contribui para identificar indivíduos no topo do quartil da distribuição, com considerável alto risco para desenvolver doenças coronarianas, os quais não podem ser considerados como indivíduos com concentrações desejáveis de lípides ${ }^{(10)}$. Os valores abaixo do ponto de corte obtido pelo P75 são considerados como aceitáveis ${ }^{(9)}$. 
O P95 de TG é adotado para definir hipertrigliceridemia, especialmente o tipo IV, de hiperlipoproteinemia, enquanto o P5 de HDL-C é utilizado para identificar indivíduos com risco de desenvolver doença cardíaca coronariana devido à relação inversa entre os níveis dessas lipoproteínas e doenças cardíacas coronarianas ${ }^{(10)}$.

A associação das dislipidemias com sobrepeso ou obesidade em crianças e adolescentes é consistente ${ }^{(5,9,11)}$. A terminologia sobrepeso é adequada para medidas antropométricas, ao passo que a obesidade refere-se a medidas de adiposidade, como a mensuração das espessuras das dobras de pele ${ }^{(17)}$.

Para avaliar sobrepeso em crianças (até nove anos de idade) optou-se pelo escore Z para peso e altura (WHZ), pois sua escala linear favorece estatística informatizada ${ }^{(17)}$ (disponível no Epi Nut/Epilnfo). Entretanto, esse recurso prejudicou a avaliação de 69 alunos que apresentaram idades e/ou medidas de altura nas extremidades superiores da curva de crescimento, sendo esses dados considerados como perdas. No grupo de adolescentes, os valores de corte do P90 para IMC estabelecidos por Sichieri e Allam ${ }^{(14)}$ para categorizar sobrepeso contribuem para adequar as categorias de sobrepeso dos alunos de Maracaí à realidade brasileira.

A prevalência de sobrepeso por método adotado em Maracaí está compatível com a expectativa ${ }^{(5)}$, e abaixo da obtida por Wang et al. ${ }^{(16)}$ que observaram 13,9\% no ano de $1997 \mathrm{em}$ crianças das regiões Nordeste e Sudeste do Brasil, seguindo os critérios da International Obesity Task Force (IOTF) ${ }^{(16)}$.

A avaliação das dislipidemias deve considerar as diferenças existentes nas concentrações de lípides e lipoproteínas entre sexos, idade e raça ${ }^{(2,9)}$. Valores absolutos de CT são consistentemente maiores no sexo feminino, em relação ao masculino, na faixa etária de 13 a 19 anos de idade ${ }^{(2)}$. Outras variáveis, que não foram objetos deste estudo, também influenciam as concentrações de lípides e lipoproteínas, como a maturação sexual( ${ }^{(4)}$ e os hábitos alimentareses ${ }^{(2,9)}$. Na puberdade, o CT no sexo masculino diminui discretamente devido à redução de $\mathrm{HDL}-\mathrm{C}$, enquanto no feminino a redução de $\mathrm{CT}$ é resultante da diminuição do $\mathrm{LDL}-\mathrm{C}^{(9)}$. As concentrações de CT são elevadas em países que mantêm dietas ricas em ácidos graxos saturados e relativamente pobres em hidratos de carbono complexos ${ }^{(2)}$.

A miscigenação e a pluralidade racial presentes na amostra estudada inviabilizaram a categorização dos participantes em relação à raça e/ou cor da pele e, conseqüentemente, os estudos sobre os pontos de corte de lípides e lipoproteínas, e as associações das dislipidemias com sobrepeso. A amostra dos alunos de Maracaí, em relação a sua constituição racial, pode ser considerada como brasileira.

Neste estudo, os valores obtidos através do P5 para o HDL-C não foram discriminativos para uma associação - que está bem estabelecida na literatura - entre valores baixos dessas lipoproteínas com sobrepeso. Uma das possíveis causas desse resultado controverso obtido em Maracaí é o pequeno número de alunos que apresentaram $\mathrm{HDL}-\mathrm{C} \leq \mathrm{P} 5$ em ambos os sexos e faixas etárias.

O ponto de corte adotado para o $\mathrm{CT} \geq \mathrm{P} 75(\geq 170 \mathrm{mg} / \mathrm{dl})$ em crianças de até nove anos de idade da cidade de Maracaí, que é o mesmo valor adotado pelas $\operatorname{DBSD}^{(11)} \mathrm{e}$ pelo $\operatorname{DPAI}^{(5)}$, não apresentou associação estatisticamente significativa com o sobrepeso em ambos sexos.

Os pontos de corte adotados para categorizar as dislipidemias, conforme os intervalos de referência estabelecidos para crianças e adolescentes de Maracaí, foram discriminativos para estabelecer associações de TG $\geq$ P95 (ambos os sexos e faixas etárias), de CT $\geq P 75$ (sexo masculino, 10-19 anos) e de LDL-C $\geq P 75$ (sexo masculino, ambas as faixas etárias) com o sobrepeso. Diversos estudos estabeleceram associações entre dislipidemias e sobrepeso ou obesidade ${ }^{(3,4,6,7,15)}$.

De acordo com Freedman et al. ${ }^{(3)}$, crianças com sobrepeso têm riscos maiores de apresentar CT $\geq 200 \mathrm{mg} / \mathrm{dl}$, LDL-C $\geq 130 \mathrm{mg} / \mathrm{dl}$ e TG $\geq 130 \mathrm{mg} / \mathrm{dl}$ (razões de chances [RC] de 2,4, 3 e 7,1, respectivamente).

Uma associação positiva obtida foi observada por Frerichs et al. entre vários níveis de obesidade com TG, LDL-C, inversa do HDL-C e, particularmente no sexo masculino, com o CT elevado ${ }^{(4)}$.

Lauer et al. (7) observaram que crianças com peso aumentado apresentaram, além de outros fatores de risco para DCV, altas concentrações de TG e baixas de HDL-C.

Adolescentes obesos apresentaram concentrações aumentadas significativas de TC $(108 \pm 54,6 \mathrm{mg} / \mathrm{dl})$, LDL-C $(119 \pm$ $28,2 \mathrm{mg} / \mathrm{dl})$, e baixas concentrações de HDL-C ( $36 \pm 9,1 \mathrm{mg} / \mathrm{dl}^{(15)}$. Em adolescentes obesos, o nível de resistência à insulina explica a variação dos níveis de TG, LDL-C e HDL-C ${ }^{(15)}$.

Nos estudos de Khoury et al.(6) as correlações de IMC foram positivas com CT, LDL-C e inversas com HDL-C. O IMC foi uma das variáveis que contribuiu significativamente para discriminação entre grupos de lipoproteínas: crianças pertencentes ao quintil superior da relação LDL-C elevado/HDL-C baixo apresentaram alto resíduo de IMC, enquanto os do quintil inferior apresentaram baixo resíduo de $\mathrm{IMC}^{(6)}$.

Apesar do tamanho da amostra ( $n$ ) de Maracaí superar o número mínimo necessário para este estudo, não pode ser desconsiderada a possibilidade de vieses decorrentes da perda de 661 alunos matriculados nas escolas da cidade, principalmente entre os adolescentes, cujos dados não puderam ser avaliados.

Este estudo fornece subsídios sobre os valores de corte dos intervalos de referência para lípides e lipoproteínas estabelecidos para crianças e adolescentes escolares de Maracaí 
e das associações das dislipidemias e sobrepeso. Devem ser consideradas as múltiplas constituições e miscigenações étnicas e os hábitos alimentares de diferentes regiões brasileiras, que podem influenciar as concentrações de lípides e lipoproteínas. Pelo fato de as doenças cardiovasculares apresentarem seu desfecho na vida adulta e de este ser um estudo transversal, sugere-se que estudos longitudinais sejam necessários para avaliar os resultados obtidos entre os participantes de Maracaí (São Paulo) e os impactos sobre sua saúde na vida adulta.

\section{Conclusão}

Os resultados apresentados fornecem subsídios para estudos sobre os intervalos de referência de lípides e lipo- proteínas para crianças e adolescentes, e permitem ampliar discussões sobre os valores dos pontos de corte adequados, que possam definir dislipidemias na população infantojuvenil brasileira.

\section{Agradecimentos}

Os autores agradecem aos alunos que participaram do estudo e seus responsáveis, aos apoios concedidos por Ortho Clinical Diagnostics (Johnson \& Johnson) pelo fornecimento dos reagentes, aos laboratórios Dr. Joelson (Assis-SP), Labmed/Labimagem (Londrina-PR) e Labmar (Maracaí-SP), e a Benedito Pereira Salatini, Claury Alves da Silva, Sandra Regina Albertini e Antonio da Silva Cavalheiro.

\section{Referências}

I. ANDRIOLO, A. Lipídeos. In: CARRAZA F.R.; ANDRIOLO, A. Diagnóstico laboratorial em pediatria. São Paulo: Sarvier; 2000. p. 99- 104

2. BROTONS, C. et al. Worldwide distribution of blood lipids and lipoproteins in childhood and adolescence: a review study Atherosclerosis, v. 139, n. I, p. 1-9, 1998.

3. FREEDMAN, D.S. et al. The relation of overweight to cardiovascular risk factors among children and adolescents: the Bogalusa Heart Study. Pediatrics, v. I03, n. 6(pt. I), p. I 175-82, 1999.

4. FRERICHS, R.R. et al. Relation of serum lipids and lipoproteins to obesity and sexual maturity in white and black children. Am J Epidemiol, v. I08, n. 6, p. 486-96, 1978.

5. GIULIANO, ICB et al. I Diretriz de Prevenção da Aterosclerose na Infância e na Adolescência. Arq Bras Cardiol, v. 85(supl. VI), p. 8-36, 2005.

6. Khoury, P. et al. Clustering and interrelationships of coronary heart disease risk factors in schoolchildren. Am J Epidemiol, v. I I 2, n 4, p.524-38, 1980.

7. LAUER, R.M.; CLARKE,W.R.; BURNS,T.L. Obesity in childhood: the Muscatine study. Acta Paed Sin, v. 38, n. 6, p. 432-7, 1997.

8. MOURA, E.C. et al. Perfil lipídico em escolares de Campinas, SP, Brasil. Rev Saúde Pública, v. 34, n. 5, p. 499-505, 2000.

9. NATIONAL CHOLESTEROL EDUCATION PROGRAM (NCEP). The Expert Panel on Blood Cholesterol Levels in Children and Adolescents. Pediatrics, v. 89(suppl. 3), p. 525-70, 1992.
10. RIFKIND, B.M.; SEGAL, P. Lipid Research Clinics Program reference values for hyperlipidemia and hypolipidemia.JAMA, v. 250, n. 14, p. 1869-72, 1983.

I I. SANTOS, R.D. III Diretrizes Brasileiras sobre Dislipidemias e Diretriz de Prevenção da Aterosclerose do Departamento de Aterosclerose da Sociedade Brasileira de Cardiologia. Arq Bras Cardiol, v. 77(supl. III), p. 4-48, 2001.

I2. SEKI, M. et al. Perfil lipídico: intervalos de referência em escolares de dois a nove anos de idade da cidade de Maracaí (SP). Bras Patol Med Lab, v. 39, n. 2, p. I 31-7, 2003.

13. SEKI, M. et al. Determinação dos intervalos de referência para lipídeos e lipoproteínas em escolares de 10 a 19 anos de idade de Maracaí (SP). J Bras Patol Med Lab, v. 39, n. 4, p. 309- 16, 2003.

14. SICHIERI, R.; ALLAM,V.L.C. Avaliação do estado nutricional de adolescentes brasileiros através do índice de massa corporal. J Pediatr, v. 72, n. 2, p. 80-4, 1996

I5. STEINBERGER, J. et al. Relationship between insulin resistance and abnormal lipid profile in obese adolescents. J Pediatr, v. 126, n. 5(pt. I), p. 690-5, 1995.

16. WANG, Y.; MONTEIRO, C.; POPKIN, B.M. Trends of obesity and underweight in older children and adolescents in the United States, Brazil, China and Russia. Am J Clin Nutr, v. 75 n. 6, p. 97I-7, 2002.

17.WORLD HEALTH ORGANIZATION (WHO). Physical status: the use and interpretation of anthropometry. World Health Organ Tech Rep Ser, v. 54. p. I-452, 1995. 